\title{
Green Communications and Networking
}

\author{
Joel J. P. C. Rodrigues \\ Instituto de Telecomunicações, University of Beira Interior \\ 6201-001 Covilhã, Portugal \\ E-mail: joeljr@ieee.org
}

Received: March 15, 2013

Accepted: March 31, 2013

Published: March 31, 2013

DOI: $10.5296 /$ npa.v5i1.3731

URL: http://dx.doi.org/10.5296/npa.v5i1.3731

\begin{abstract}
This Special Issue includes the best revised selected papers from The Second International Conference on Green Communications and Networking (GreeNets 2012), that took place in Gandia, Valencia, Spain, at October 25-26, 2012. This second edition of GreeNets received very good contributions. Then, this special issue includes the better conference papers after a rigorous review process in order to revise and enlarge the original contributions while avoiding self-plagiarism.
\end{abstract}

Keywords: Green Communications, Communications and Networking, Energy-efficient network architecture \& protocols, Systems and Technology, Energy-efficient management. 


\section{Introduction}

Global warming and climate change have been a growing worldwide concern. Six sources, i.e., transportation, power, buildings, industry, agriculture and forestry, and land use, have been identified as major contributors to the rise of global carbon dioxide (CO2). The mobile industry is seen as a potential enabler to reduce greenhouse gases contributed by these six sources provided that appropriate measures are implemented. On the other hand, the mobile industry itself will also contribute to $\mathrm{CO} 2$ emission through network operations, mobile equipment, etc. To meet the requirement of low-carbon economy development, it is necessary to reduce the operation expenditure or energy consumption of mobile networks, while maintaining acceptable quality of service. This special issue explores and explains the scope and challenges of designing, building, and deploying GreeNets. In this regard, this special issue considers high-level contributions considering different topics including green mobile networks, system architectures, networking \& communication protocols, applications, test-bed and prototype, traffic balance and energy-efficient cooperation transmission, system and application issues related to GreeNets.

Along its four papers, protocols and algorithms solutions are presented, furnishing important contributions to the state of the art and offering, at the same time, an important updated overview about emerging communication technologies for Green Communications and Networks.

The paper entitled "EE-ARQ: a Green ARQ-Based Algorithm for the Transmission of Video Streams on Noise Wireless Channels" [1], proposed by Alfio Lombardo, Carla Panarello, and Giovanni Schembra addresses an important issue related with the increase of the energy efficiency of telecommunications networks, and specifically wireless devices that present the highest energy consumption coefficient per bit transmitted among all the networking devices. However, making a network device green can cause performance deterioration. The target of this paper is to propose a new algorithm for the transmission of multiplexed rate-controlled multimedia streams over wireless channels. The algorithm is an energy-efficient variant of ARQ to exploit the correlation of the wireless channel behavior. In addition, in order to compensate transmission bandwidth reduction due to the energy saving policies, a cross-layer approach is applied introducing a source Rate Controller working to modify the video quality according to the state of the transmission buffer. An analytical model of the whole system is presented in order to evaluate performance and provide some guidelines to design the configuration parameters of the proposed algorithm.

The paper by Keiko Karaishi and Masato Oguchi, "Development and Evaluation of Smart Grid Simulation System with Power Stabilization by EV" [2], proposes a simulation system developed as a method to evaluate power control when electrical vehicles (EVs) are connected to the Smart Grid as a power source. First, a power grid simulation environment will be constructed, and the impact of EVs connected to the power grid in this simulated environment will be evaluated. Specifically, authors connect many EVs to the power grid environment, discharged the batteries, and monitor the voltage fluctuations at each point. Then, some algorithms were proposed to determine which EV should be discharged, and 
evaluate efficacy of electric power stabilization. As a result of the evaluation, the proposed and developed system works to provide means of a useful evaluation for various cases of the Smart Grid.

The paper by Augusto Morales Domínguez, Tomas Robles, Ramon Alcarria, and Edwin Cedeño, "A Hot-topic based Distribution and Notification of Events in Pub/Sub Mobile Brokers" [3], introduce mechanisms that allow mobile brokers to distribute their sub-scribers and coordinate the notification of events between fixed and mobile brokers. The authors propose a hot-topic algorithm that marks the event popularity and depending on this, delegates or recovers subscriptions. Furthermore, they also propose extensions for the MQTT protocol, in order to support our mechanisms. Finally, the results performed through simulation confirm that proposed mechanisms offer advantages for the considered Internet of Things (IoT) scenario. The results show that proposed Hot-Topic algorithm improves the publication delay whenever a RMB delegates the notification of events to the fixed broker, in comparison with a naïve approach. They also confirm that used sliding window can be used for adapting the algorithm to different set of traffic, fact that increases the pluggability of mobile brokers in unknown scenarios. In random traffic the performance of the hot-topic algorithm is similar to the naïve approach, so using the hot-topic algorithm neither improves nor worsens the publication de- lay.

The paper presented by Sami J. Habib and Paulvanna N. Marimuthu, "Comparing Communication Protocols within an Enterprise Network for Carbon Footprint Reduction" [4], presents a comparison study between transport-layer communication protocols relative to carbon footprint within enterprise network. The comparative study focuses on three factors: the transport layer communication protocols (TCP and UDP), the QoS offered by the transmission line, and the data encoding schemes in the physical layer. The QoS of the transmission line and the number of transmissions contribute significantly to the total carbon footprint generated by the enterprise network. The carbon footprint is estimated from the power spectrum of the transmitted packets through Manchester coding. The carbon reduction at the backbone is of prime importance, since the enterprise is often susceptible to heavy transactions at the backbone. The authors have formulated the carbon footprint reduction within the enterprise as an optimization problem, wherein the given single enterprise is synthesized into suitable clusters by localizing the heavily communicating nodes together. The simulation results demonstrate that a typical single enterprise comprising of 100 nodes with 4 GB generated backbone traffic when both the UDP and TCP utilize high capacity link, produce low and nearly equal volumes of carbon. However, the difference becomes significantly high with the link offering poor QoS, in which, UDP based transmissions produce $14 \%$ less carbon than TCP based transmissions. The optimization within the molecular assembly algorithm manages to produce $64.5 \%$ reduced annual carbon emission than the initial network.

We believe that this special issue provides a better understanding of advances on network Protocols \& Algorithms for green communications and networking. To conclude this special Issue, we would like to thank all the authors that submitted papers for this special Issue and for leveraging the quality of this publication. We would also like to thank the reviewers for 


\section{Macrothink}

their timely comprehensive reviews and constructive comments. Finally, we wish to express our thanks to Jaime Lloret Mauri, Editor-in-Chief of Network Protocols and Algorithms, for his help and cooperation on this publication.

\section{References}

[1] Alfio Lombardo, Carla Panarello, and Giovanni Schembra, "EE-ARQ: a Green ARQ-Based Algorithm for the Transmission of Video Streams on Noise Wireless Channels", Network Protocols and Algorithms, Vol. 5, Issue 1, pp. 41-70, 2013. http://dx.doi.org/10.5296/npa.v5i1.3180

[2] Keiko Karaishi and Masato Oguchi, "Development and Evaluation of Smart Grid Simulation System with Power Stabilization by EV", Network Protocols and Algorithms, Vol. 5, Issue 1, pp. 71-89, 2013. http://dx.doi.org/10.5296/npa.v5i1.3261

[3] Augusto Morales Domínguez, Tomas Robles, Ramon Alcarria, and Edwin Cedeño, "A Hot-topic based Distribution and Notification of Events in Pub/Sub Mobile Brokers", Network Protocols and Algorithms, Vol. 5, Issue 1, pp. 90-110, 2013. http://dx.doi.org/10.5296/npa.v5i1.3326

[4] Sami J. Habib and Paulvanna N. Marimuthu, "Comparing Communication Protocols within an Enterprise Network for Carbon Footprint Reduction", Network Protocols and Algorithms, Vol. 5, Issue 1, pp. 111-126, 2013. http://dx.doi.org/10.5296/npa.v5i1.3342

\section{Copyright Disclaimer}

Copyright reserved by the author(s).

This article is an open-access article distributed under the terms and conditions of the Creative Commons Attribution license (http://creativecommons.org/licenses/by/3.0/). 